\title{
Erratum to: Apoptosis index correlates with chemotherapy efficacy and predicts the survival of patients with gastric cancer
}

Yongning Jia, Bin Dong, Lei Tang, Yiqiang Liu, Hong Du, Peng Yuan, Aiwen Wu and Jiafu Ji [Tumor Biology, 33, 2012, 1151-1158. DOI: 10.1007/s13277-012-0357-8]

In a recent review, the authors identified an error in the figure of apoptotic index and pathological response grade in Fig. 2 of the published article. The corrected figure is given below. This error did not affect either the results or the conclusions reported in the paper. The authors apologize to the readers of Tumor Biology for any inconvenience caused.

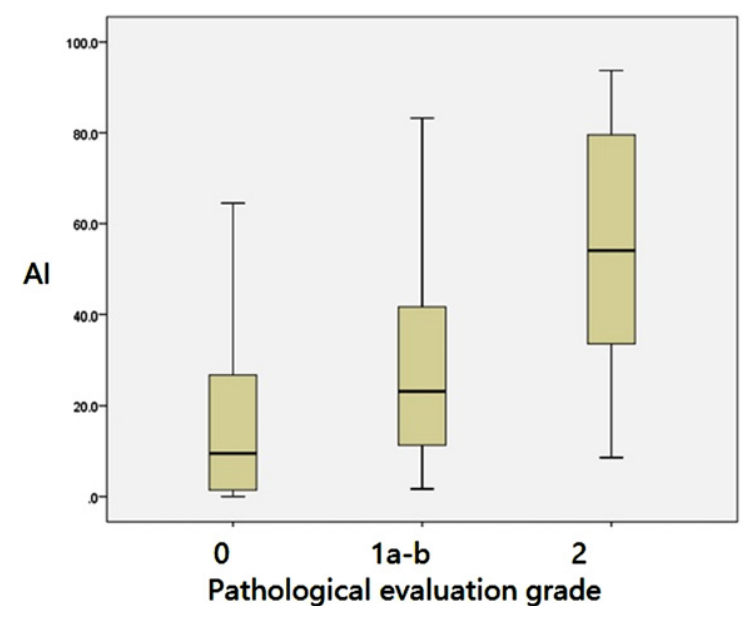

